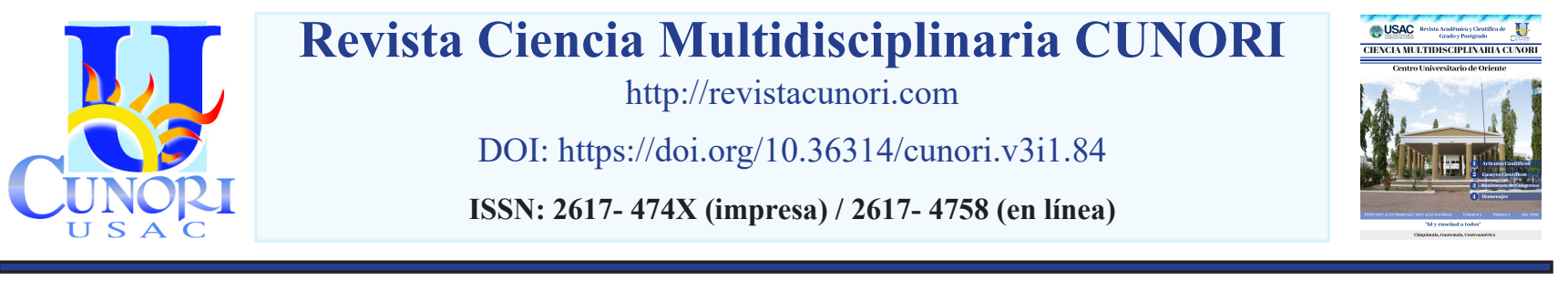

Como citar el artículo

Reyes, J., Mazariegos, E., Arriola, C., Vides, R., Sosa, C., (2019). Riesgo epidemiológico de desarrollar diabetes mellitus tipo 2. Revista Ciencia Multidisciplinaria CUNORI, 3(1), 93-99. DOI: https://doi.org/10.36314/cunori.v3i1.84

\title{
Riesgo epidemiológico de desarrollar diabetes mellitus tipo 2
}

\section{Epidemiologic risk of developing diabetes type 2}

Julio Reyes*, Edvin Mazariegos, Carlos Arriola, Rory Vides. Christian Sosa Centro Universitario de Oriente (CUNORI), Universidad de San Carlos de Guatemala

Recibido: 27 de agosto de 2018 / Revisión: 29 de septiembre de 2018 / Aceptado: 23 de enero de 2019

Disponible en internet el 30 de agosto de 2019

$\begin{array}{ll} & * \text { Autor para correspondencia. } \\ \text { Resumen } & \text { Correo electrónico: julioadan1@hotmail.com }\end{array}$

E 1 riesgo de padecer diabetes se viene estudiando desde que se descubrió dicha patología. En Europa se ha utilizado el test FIN$\triangle$ DRISC para tamizar a los pacientes con alto riesgo de desarrollar diabetes. Estudio descriptivo transversal que se realizó en 374 pacientes escogidos mediante muestreo probabilístico, aleatorio simple, que acudieron al Centro de Salud de San Jacinto, Chiquimula. El objetivo fue evaluar el nivel de riesgo de desarrollar diabetes mellitus tipo 2 dentro de 10 años en la población a través del test FINDRISC. El nivel de riesgo para desarrollar diabetes de la población más frecuente fue el riesgo ligeramente elevado con $29.4 \%(n=110)$, el riesgo alto con $25.2 \%(n=94)$ y el riesgo muy alto con $7.5 \%(n=28)$, el sexo más afectado fue el femenino con $73 \%(n=69)$ de casos en riesgo alto y $82 \%(n=23)$ casos de riesgo muy alto, el grupo etario más afectado para un riesgo alto fueron los menores de 45 años con 34\%(n=32) y para un riesgo muy alto, entre 55 a 64 años con 36\% (n=10). Las 3 causas más frecuentes con riesgo alto de desarrollar diabetes, fueron: la falta de consumo de frutas y/o verduras con 35\% ( $\mathrm{n}=33$ ), falta de actividad física durante 30 minutos al día, con 33\% ( $\mathrm{n}=31)$ y un nivel alto de glucosa en el control médico (prediabetes) con 32\% ( $\mathrm{n}=30$ ). Para el riesgo muy alto, la herencia predominó con $35.7 \%(n=10)$, seguido de un nivel alto de glucosa con $35.7 \%$ ( $n=10)$, y la falta de actividad física con $28.6 \%(\mathrm{n}=8)$. Se determinó que 59 pacientes de la muestra desarrollarán diabetes por lo que, de 13,089 pobladores, el 15.96\% $(n=2,089)$ la desarrollarán dentro de 10 años, datos calculados a partir de resultados obtenidos del test FINDRISC.

Palabras clave: FINDRISC, diabetes mellitus tipo 2, riesgo, glucosa, test

\section{Abstract}

$\mathrm{T}$ he risk of suffering from diabetes has been studied since this pathology was discovered. In Europe, the FINDRISC test has been used to screen patients at high risk of developing diabetes. Transverse descriptive study in 374 patients were chosen by simple randomized probabilistic sampling, who attended the Health Center of San Jacinto, Chiquimula. The objective was to evaluate the level of risk of developing type 2 diabetes mellitus within 10 years in the population through the FINDRISC test. The risk level to develop diabetes of the most frequent population was the slightly elevated risk with $29.4 \%(\mathrm{n}=110)$, the high risk with $25.2 \%$ ( $\mathrm{n}$ $=94)$ and the very high risk with $7.5 \%(n=28)$, the sex most affected was the female with $73 \%(n=69)$ of cases at high risk and $82 \%(n=23)$ cases of very high risk, the age group most affected for a high risk were those under 45 with $34 \%(n=32)$ and for a very high risk, between 55 to 64 years with $36 \%(\mathrm{n}=10)$. The 3 most frequent causes with high risk of developing diabetes were: lack of fruit and / or vegetable consumption with $35 \%(n=33)$, lack of physical activity for 30 minutes a day, with $33 \%(n=31)$ and a high glucose level in medical control (prediabetes) with $32 \%(\mathrm{n}=30)$. For very high risk, inheritance predominated with $35.7 \%(\mathrm{n}=10)$, followed by a high glucose level with $35.7 \%(\mathrm{n}=10)$, and lack of physical activity with $28.6 \%(\mathrm{n}=8)$. It was determined that 59 patients in the sample developed diabetes, so that, from 13,089 inhabitants, $15.96 \%(\mathrm{n}=2,089)$ developed it within 10 years, data calculated from the results obtained from the FINDRISC test.

Keywords: FINDRISC, type 2 diabetes mellitus, risk, glucose, test 


\section{Introducción}

El riesgo de padecer diabetes mellitus se viene estudiando desde el mismo tiempo que se descubrió dicha patología, se ha asociado a factores genéticos y a estilos de vida, como es la inactividad física, el sobrepeso, la circunferencia abdominal, el IMC, la edad y una ingesta alta en carbohidratos, la identificación de dichos factores es esencial para su prevención y detección precoz por lo que se han realizado estudios para crear herramientas fácilmente reproducibles y económicas, entre éstas se encuentra el test Finlandés FINDRISC que se viene validando desde 1992 en Finlandia y España el cual se utilizó en esta investigación (Lindström \& Tuomilehto, 2003:725-731).

Se evaluaron 8 parámetros tanto de interrogatorio (familiares con diabetes, edad, actividad física, consumo de frutas/verduras y consumo de medicamentos para hipertensión) así como físicos (IMC, perímetro abdominal) y bioquímicos (glucosa en sangre capilar) para determinar el riesgo de la población que más está afectada con diabetes mellitus en el departamento de Chiquimula y se desecharon los casos de pacientes diabéticos debutantes por no ser parte de los objetivos de dicho estudio (ADM, 2013:01).

Con los datos de la presente investigación se determinó que el nivel de riesgo que más presento la población fue el riesgo ligeramente elevado, pero con un alarmante numero el riesgo alto y muy alto. Los factores predominantes que evaluó el test son los del tipo modificables: alimentación balanceada y ejercicio ligados en menor cantidad a la herencia. Además, se determinó que el sexo más afectado es el femenino en ambos niveles de riesgo, muchas veces el sedentarismo producto de dedicarse ampliamente al oficio doméstico y a la mayor cantidad que acude al Centro de Salud, produjo estos resultados. Este estudio tiene como objetivo determinar el riesgo de la población de San Jacinto Chiquimula mediante una muestra de pacientes que asistieron al Centro de Salud de dicha región en los meses de junio y julio del año 2018.

\section{Material y métodos}

Se realizó un estudio descriptivo transversal con una muestra probabilística, aleatoria simple de 374 pobladores del municipio de San Jacinto, Chiquimula, pertenecientes a todas sus aldeas, que asisten al Centro de Salud, de una población de 13,089 personas. Se excluyen los pacientes que no pertenecen o residen en el municipio, con diagnóstico previo de diabetes mellitus o con criterios diagnósticos de diabetes mellitus por glucometría al momento del estudio.

Todos los pacientes fueron evaluados con los mismos criterios, se les informó de las características y objetivos del estudio y tras su aceptación mediante consentimiento informado se procedió a una anamnesis la cual incluyó, edad, consumo de frutas y/o verduras, familiares con diabetes mellitus, toma de medicamentos para la hipertensión, actividad física y medición de parámetros físicos como es el perímetro abdominal al nivel del ombligo y el índice de masa corporal (IMC) mediante la toma del peso y la talla y un parámetro de laboratorio como es la glicemia capilar en glucómetro portátil tras un ayuno mínimo de 8 horas para detectar prediabetes y se anotó en el test de FINDRISC.

El puntaje de riesgo de diabetes como se le denomina al test de FINDRISC se compone de una suma de puntajes individuales en un rango de 0 a 26, mientras más alto sea el puntaje obtenido mayor será 
la probabilidad de desarrollar diabetes entre los próximos 10 años: $<7$ puntos, bajo riesgo; de 7 a 11 puntos, ligeramente elevado; de 12 a 14 puntos, moderado; de 15 a 20 puntos, alto y más de 20 puntos, riesgo muy alto. Los criterios para diagnosticar prediabetes fueron una glicemia con un mínimo de 8 horas de ayuno con valores de $\geq 100 \mathrm{a} \leq 125 \mathrm{mg} / \mathrm{dl}$ e IMC con valores menores de $25 \mathrm{~kg} / \mathrm{m} 2$ para bajo peso, entre 25 y $30 \mathrm{~kg} / \mathrm{m} 2$ para el sobrepeso y mayor a $30 \mathrm{~kg} / \mathrm{m} 2$ para la obesidad (López, 2016:18-24).

Para los niveles de muy alto riesgo se determinó según el test que 1 de cada 2 desarrollarán diabetes mellitus dentro de 10 años; para el nivel de riesgo alto 1 de cada 3, nivel de riesgo moderado 1 de cada 6, ligeramente elevado 1 de cada 25, bajo 1 de cada 100 pacientes (Fornos, et al. 2013:141-146).

\section{Resultados}

\begin{tabular}{|l|c|c|}
\hline FINDRISC & $\mathbf{N}=\mathbf{3 7 4}$ & $\begin{array}{c}\text { Porcentaj } \\
\text { e (100\%) }\end{array}$ \\
\hline Bajo riesgo & $\mathrm{n}=88$ & $23.50 \%$ \\
\hline $\begin{array}{l}\text { Riesgo } \\
\text { ligeramente } \\
\text { elevado }\end{array}$ & $\mathrm{n}=110$ & $29.40 \%$ \\
\hline $\begin{array}{l}\text { Riesgo } \\
\text { moderado }\end{array}$ & $\mathrm{n}=54$ & $14.40 \%$ \\
\hline Riesgo alto & $\mathrm{n}=94$ & $25.20 \%$ \\
\hline $\begin{array}{l}\text { Riesgo muy } \\
\text { alto }\end{array}$ & $\mathrm{n}=28$ & $7.50 \%$ \\
\hline
\end{tabular}

Cuadro 1. Distribución de los pacientes que asistieron al Centro de Salud de San Jacinto, Chiquimula, según el nivel de riesgo para desarrollar diabetes mellitus tipo 2, determinado por el test FINDRISC.

El nivel de riesgo de la población más frecuente fue el de riesgo ligeramente elevado con $29.4 \%$ ( $\mathrm{n}=110)$ pero cabe resaltar el riesgo alto con $25.2 \%(\mathrm{n}=94)$ y con una importante cantidad el riesgo muy alto con $7.5 \%(n=28)$.

\begin{tabular}{|l|c|l|c|}
\hline FINDRISC & Masculino & Femenino & $\begin{array}{c}\text { Totales: } \\
\mathbf{N = 1 2 2}\end{array}$ \\
\hline Riesgo alto & $27 \%(n=25)$ & $73 \%(n=69)$ & $\begin{array}{c}100 \% \\
(n=94)\end{array}$ \\
\hline $\begin{array}{l}\text { Riesgo muy } \\
\text { alto }\end{array}$ & $18 \%(n=5)$ & $82 \%(n=23)$ & $\begin{array}{c}100 \% \\
(n=28)\end{array}$ \\
\hline
\end{tabular}

Cuadro 2. Distribución de pacientes que asistieron al Centro de Salud de San Jacinto, Chiquimula, con riesgo alto y muy alto de desarrollar diabetes mellitus tipo 2, según el sexo por medio del test FINDRISC.

El sexo femenino tuvo predominio con $73 \%(n=69)$ en el nivel de riesgo alto y $82 \%(n=23)$ en el muy alto, mientras que el masculino con $27 \%(n=25)$ en riesgo alto y $18 \%(n=5)$ en el muy alto por lo que se evidencia una diferencia marcada a favor del sexo femenino sobre el masculino. 


\begin{tabular}{|c|c|c|c|}
\hline $\begin{array}{c}\text { Edad } \\
\text { (años) }\end{array}$ & Riesgo alto & $\begin{array}{c}\text { Riesgo muy } \\
\text { alto }\end{array}$ & Frecuencia \\
\hline$\leq 45$ & $34 \%(n=32)$ & $14 \%(n=4)$ & $30 \%(n=36)$ \\
\hline 45 a 54 & $19 \%(n=18)$ & $25 \%(n=7)$ & $20 \%(n=25)$ \\
\hline 55 a 64 & $22 \%(n=21)$ & $36 \%(n=10)$ & $25 \%(n=31)$ \\
\hline$\geq 64$ & $25 \%(n=23)$ & $25 \%(n=7)$ & $25 \%(n=30)$ \\
\hline Totales & $\begin{array}{c}100 \% \\
(n=94)\end{array}$ & $\begin{array}{c}100 \% \\
(n=28)\end{array}$ & $\begin{array}{c}100 \% \\
(N=122)\end{array}$ \\
\hline
\end{tabular}

Cuadro 3. Distribución de pacientes que asistieron al Centro de Salud de San Jacinto, Chiquimula, con riesgo alto y riesgo muy alto de desarrollar diabetes mellitus tipo 2, según la edad por medio del test FINDRISC.

Se determinó la frecuencia de los niveles de riesgo por grupos etarios, predominando el grupo de edad $\leq 45$ en riesgo alto con $34 \%(n=32)$ y entre 55 a 64 años en riesgo muy alto con $36 \%(n=10)$.

\begin{tabular}{|l|c|c|c|c|}
\hline $\begin{array}{c}\text { Causas más } \\
\text { frecuentes }\end{array}$ & $\begin{array}{c}\text { Falta de } \\
\text { consumo de } \\
\text { frutas y/o } \\
\text { verduras } \\
\text { diariamente }\end{array}$ & $\begin{array}{c}\text { Falta de } \\
\text { actividad } \\
\text { física } \\
\text { durante 30 } \\
\text { minutos al } \\
\text { día }\end{array}$ & $\begin{array}{c}\text { Nivel alto de } \\
\text { glucosa en } \\
\text { control } \\
\text { médico } \\
\text { (prediabetes) }\end{array}$ & Totales \\
\hline Riesgo alto & $35 \%(n=33)$ & $33 \%(n=31)$ & $32 \%(n=30)$ & $\begin{array}{c}100 \% \\
(N=94)\end{array}$ \\
\hline
\end{tabular}

Cuadro 4. Distribución de pacientes que asistieron al Centro de Salud de San Jacinto, Chiquimula, según las 3 causas más frecuentes que presentaron, en el nivel de riesgo alto de desarrollar diabetes mellitus tipo 2, por medio del test FINDRISC.

Para el nivel de riesgo alto se estableció que la falta de consumo de frutas y/o verduras diariamente fue la causa predominante con 35\%, seguido por la falta de actividad física durante 30 minutos al día con $33 \%$ y un nivel alto de glucosa (prediabetes) en un control médico con 32\% de los casos. Siendo éstas las 3 causas que más afectaron a los pacientes con riesgo alto de desarrollar diabetes tipo 2 en 10 años. 


\begin{tabular}{|l|c|c|c|c|}
\hline $\begin{array}{c}\text { Causas más } \\
\text { frecuentes }\end{array}$ & $\begin{array}{c}\text { Diagnóstico } \\
\text { en } \\
\text { familiares } \\
\text { con } \\
\text { diabetes } \\
\text { (padres, } \\
\text { hijos y/o } \\
\text { hermanos) }\end{array}$ & $\begin{array}{c}\text { Nivel alto de } \\
\text { glucosa en } \\
\text { control médico } \\
\text { (prediabetes). }\end{array}$ & $\begin{array}{c}\text { Falta de } \\
\text { actividad } \\
\text { física } \\
\text { durante 30 } \\
\text { minutos al } \\
\text { día }\end{array}$ & Totales \\
\hline $\begin{array}{l}\text { Riesgo muy } \\
\text { alto }\end{array}$ & $35.7 \%(n=10)$ & $35.7 \%(n=10)$ & $28.6 \%(n=8)$ & $\begin{array}{c}100 \% \\
(N=28)\end{array}$ \\
\hline
\end{tabular}

Cuadro 5. Distribución de pacientes que asistieron al Centro de Salud de San Jacinto, Chiquimula, según las 3 causas más frecuentes que presentaron, en el nivel de riesgo muy alto de desarrollar diabetes mellitus tipo 2, por medio del test FINDRISC.

En el nivel de riesgo muy alto las causas predominantes fueron los antecedentes familiares con diagnóstico de diabetes con $35.7 \%$ de casos, al igual que el nivel alto de glucosa en control médico (prediabetes) que obtuvo $35.7 \%$ casos seguido de la falta de actividad física durante 30 minutos al día con $28.6 \%$. Siendo éstas las 3 causas que más afectaron a los pacientes con riesgo muy alto de desarrollar diabetes tipo 2 en 10 años.

\section{Discusión}

El test determinó los siguientes resultados: de las 374 personas que acudieron al Centro de Salud 28 presentaron riesgo muy alto de desarrollar diabetes mellitus a 10 años, un $7.5 \%$ por lo que 14 desarrollarán la enfermedad, 94 con riesgo alto para un $25.2 \%$ por lo que 31 pobladores desarrollarán la enfermedad, riesgo moderado con 54 para un $14.4 \%$ por lo que 9 desarrollarán la enfermedad, riesgo ligeramente elevado con 110 para un $29.4 \%$ por lo que 4 desarrollarán la enfermedad, bajo riesgo 88 para un $23.5 \%$ por lo que menos de 1 persona desarrollara la enfermedad para un total de 59 pacientes que desarrollarán diabetes mellitus tipo 2 a 10 años según mediante el test de FINDRISC (Cuadro 1).

A nivel poblacional San Jacinto posee 13,089 pobladores obteniéndose los siguientes resultados a partir de la muestra: un riesgo muy alto con $7.5 \%$ por lo que 490 pobladores desarrollarán diabetes mellitus, riesgo alto con $25.2 \%$ por lo que 1,099 desarrollarán la enfermedad, riesgo moderado con $14.4 \%$ por lo que 314 personas desarrollarán la enfermedad, riesgo ligeramente elevado con $29.4 \%$ por lo que 154 desarrollarán la enfermedad y bajo riesgo con $23.5 \%$ por lo que 31 desarrollarán la enfermedad para un total de 2,089 que desarrollarán diabetes mellitus tipo 2, esto equivale a un $15.96 \%$ de la población una cantidad que corrobora el hecho que haya presentado un número elevado de pacientes debutantes en este municipio (Centro de Salud San Jacinto, 2017:01).

El sexo femenino evidenció un mayor riesgo de desarrollar diabetes mellitus tipo 2 en 10 años después, con una relación aproximada de 3:1 con el sexo masculino. El grupo etario que presentó más riesgo de desarrollar diabetes mellitus a 10 años con el $30 \%$ de los casos fueron los pacientes menores de 45 años, seguido por el grupo entre 55 y 64 años con $25 \%$ de los casos. 
Las 3 causas más frecuentes de desarrollar diabetes mellitus en el grupo de estudio con riesgo alto fueron: Falta de consumo de frutas y/o verduras diariamente con $36 \%$, falta de actividad física durante 30 minutos al día con 33\% y un nivel alto de azúcar (glucosa) en un control médico con 32\% (prediabetes).

Las 3 causas más frecuentes de desarrollar diabetes mellitus en el grupo de estudio con riesgo muy alto fueron: Diagnóstico de familiares con diabetes (Padres, hijos y/o hermanos) con 35.7 \%, nivel alto de azúcar (glucosa) en un control médico (prediabetes) con $35.7 \%$ y falta de actividad física durante 30 minutos con $28.6 \%$.

En relación a la detección de la diabetes mellitus se ha utilizado criterios recomendados para las pruebas de cribado, entre estas se encuentra la aceptabilidad (mientras más sencillas, rápidas y económicas es mejor), validez (sensibilidad y especificidad de la prueba), fiabilidad (la repetición de la prueba en las mismas condiciones ofrece los mismos resultados) por lo que el test cumple con estas especificaciones. Otro de los aspectos a tomar en cuenta es que el test para el cribado de diabetes debe tener ciertas consideraciones con el objetivo de detectar pacientes con diabetes mellitus en este caso tres pruebas (glucosa en ayunas, postprandial y hemoglobina glicosilada) son válidas para detectar un riesgo aumentado de padecer diabetes tipo 2 (Puente, 2015:34-35).

\section{Agradecimientos}

Este trabajo ha sido posible gracias al apoyo y asesoría de las autoridades de la carrera de médico y cirujano del Centro Universitario de Oriente (CUNORI), al Dr. Edvin Danilo Mazariegos, a la Licenciada en Ciencias de la Comunicación Rosalía Marisol Reyes y al personal del Centro de Salud del municipio de San Jacinto.

\section{Referencias bibliográficas}

Asociación Diabetes Madrid ADM. (2013). El test de FINDRISC, una herramienta que te indica el riesgo de padecer diabetes tipo 2. España. Recuperado de https://diabetesmadrid.org/ el-test-de-findrisc-un-herramienta-que-te-indica-el-riesgo-de-padecer-diabetes/

Centro de Salud San Jacinto. (2017). Memoria de estadísticas vitales y vigilancia epidemiológica: indicadores básicos de análisis de situación de salud república de Guatemala (programa de computo Excel). San Jacinto, Chiquimula, Guatemala.

Fornos, J.et al. (2013). Detección de pacientes con riesgo de desarrollar diabetes en farmacias comunitarias de Pontevedra. Revista Farmacéuticos Comunitarios 5(4). Recuperado de http:/www.farmaceuticoscomunitarios.org/es/system/files/ journals/574/articles/5-4-141-146.pdf

Lindström, J. \& Tuomilehto, J. (2003). The diabetes risk score: a practical tool to predict type 2 diabetes risk. Revista Diabetes Care 26(3). https://doi.org/10.2337/ diacare.26.3.725

López, T. (2016). Factores de riesgo de diabetes tipo 2 entre los colaboradores de una compañía de seguridad de la ciudad de Guayaquil. Tesis Lic. Samborondón, Ecuador, UEES. Recuperado de http:// repositorio.uees.edu.ec/bitstream/123456789/ 1930/1/Tannya Lopez Trabajo de Titulación UEES 2016.pdf 
Puente, D. (2015:). ¿Cuál es la prueba más fiable para el cribado de la diabetes ¿glucemia en ayunas, sobrecarga oral de la glucosa o hemoglobina glucosilada? In Guía de actualización en diabetes mellitus tipo 2. España, Editorial Euromedice Vivactis. Recuperado de http://www.redgdps.org/gestor/ upload/GUIA2016/Guia_Actualizacion_2016v2.pdf

\section{Sobre el autor}

\section{Julio Adan Reyes López}

Médico y Cirujano, egresado del Centro Universitario de Oriente CUNORI, de la Universidad de San Carlos de Guatemala. Ha realizado investigaciones sobre "Efectos de la rosuvastatina y atorvastatina sobre lipoproteinas de baja densidad en población Chiquimulteca", "Complicaciones maternas en embarazos de adolescentes", "Nivel de conocimientos acerca de reanimacion neonatal avanzada en estudiantes de medicina del hospital nacional Carlos Manuel Arana Osorio", "Incidencia de infecciones asociadas a servicios de salud", "Evaluacion de las maniobras de Leopold", "Secuelas a mediano plazo en pacientes diagnosticados con CHIKV por laboratorio y nexo epidemiologico en la aldea San Esteban". Su más reciente investigación es el "Riesgo Epidemiológico de desarrollar diabetes mellitus tipo 2".

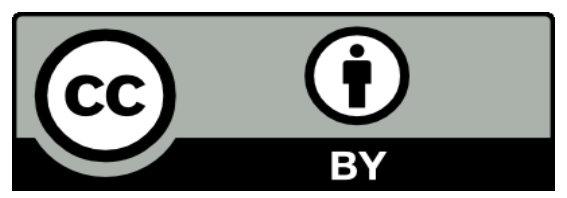

Este texto está protegido por una licencia CreativeCommons 4.0.

Usted es libre para compartir, copiar y redistribuir el material en cualquier medio o formato y adaptar el documento, remezclar, transformar y crear a partir del material para cualquier propósito, incluso comercialmente, siempre que cumpla la condición de atribución: usted debe reconocer el crédito de una obra de manera adecuada, proporcionar un enlace a la licencia, e indicar si se han realizado cambios. Puede hacerlo en cualquier forma razonable, pero no de forma tal que sugiera que tiene el apoyo del licenciante o lo recibe por el uso que hace. 